\title{
The Role of MR Imaging in Assessment of Brain Damage from Carbon Monoxide Poisoning: A Review of the Literature
}

T. Beppu

\begin{abstract}
SUMMARY: The aim of this article is to review how MR imaging and associated imaging modalities provide clinicopathologic information on brain damage after carbon monoxide poisoning. Initially, many authors documented typical findings of conventional MR imaging in the gray matter structures such as the globus pallidus and in various regions of cerebral white matter. The focus of investigation has since shifted to observation of cerebral white matter areas that are more frequently detected on MR imaging and are more responsible for chronic symptoms than the gray matter. DWI has dramatically contributed to the ability to quantitatively assess cerebral white matter damage. Subsequently, DTI has enabled more sensitive evaluation than DWI and can demonstrate progressive pathologic changes in the early stage, allowing prediction of chronic conditions. In addition, MR spectroscopy reveals changes in metabolite levels, offering quantitative clinicopathologic information on brain damage after carbon monoxide poisoning.
\end{abstract}

ABBREVIATIONS: $C O$ = carbon monoxide; $C W M=$ cerebral white matter; DNS = delayed neuropsychiatric sequelae; $F A=$ fractional anisotropy; $G P=$ globus pallidus; $\mathrm{MBP}=$ myelin basic protein

O poisoning causes serious damage to the brain and cardiac muscles. Damage to the brain directly affects the prognosis for patients who survive CO poisoning. Some clinical obstacles exist to the assessment of brain damage among such patients. First, predicting clinical behaviors such as specific types of chronic neuropsychiatric symptoms is difficult. Surviving patients will display 1 of 3 clinical behavioral types in the chronic phase after CO poisoning: approximately $70 \%$ of survivors present with various transient symptoms only in the acute phase; $20 \%$ of patients present with symptoms persisting from the acute to the chronic phase; and the remaining 10\% exhibit DNS, representing recurrent neuropsychiatric symptoms occurring after an interval of apparent normality (the so-called lucid interval; mean duration, 22 days) after the apparent resolution of acute symptoms. ${ }^{1-5}$ Acute conditions including level of consciousness and carboxyhemoglobin concentration have been considered and found to be unhelpful in the prediction of chronic clinical behaviors. ${ }^{6,7}$ In particular, predicting whether patients who exhibit resolved acute symptoms have escaped or will experience DNS represents a very important clinical issue. ${ }^{8}$ A second problem is the difficulty of

From the Department of Neurosurgery, Department of Hyperbaric Medicine, Iwate Medical University, Morioka, Japan.

Please address correspondence to Takaaki Beppu, MD, PhD, Department of Neurosurgery, Department of Hyperbaric Medicine, Iwate Medical University, Morioka, Japan., 19-11, Uchimaru, Morioka, 020-8505 Japan; e-mail: tbeppu@iwate-med.ac.jp

- Indicates open access to non-subscribers at www.ajnr.org

http://dx.doi.org/10.3174/ajnr.A3489 assessing the severity of brain damage. Chronic symptoms include parkinsonism, dystonia or other motor impairments, cognitive or executive dysfunction, akinetic mutism, mood disorder, and personality change. The severity of brain damage cannot be directly compared among patients with different symptoms. One of the strategies to resolve these clinical problems is to apply objective assessments of brain damage by using MR imaging, a readily available and minimally invasive procedure. Here, I review the literature on assessment of brain damage secondary to CO poisoning by use of conventional MR imaging in addition to other sequences, such as DWI, DTI, and MR spectroscopy, and summarize what MR imaging can currently accomplish in the assessment of brain damage after CO poisoning.

\section{MECHANISMS CAUSING BRAIN DAMAGE AFTER CO EXPOSURE}

Comprehension of the mechanisms causing brain damage after $\mathrm{CO}$ exposure is crucial to understanding the findings of MR imaging in the assessment of brain damage. Brain damage has been considered to result from complicated mechanisms, as follows. ${ }^{4}$ The uptake of CO by hemoglobin with an affinity 200 times that of oxygen leads to hypoxia in tissues, and a shift in the oxyhemoglobin dissociation curve to the left inhibits release of oxygen, further exacerbating tissue hypoxia. ${ }^{9}$ Whereas hypotension adds to hypoxia, the hypoxic-hypotension process leads to ischemic changes in the arterial border zones in the brain. ${ }^{10}$ Brain hypoxia increases the level of excitatory amino acids including glutamine, leading to 


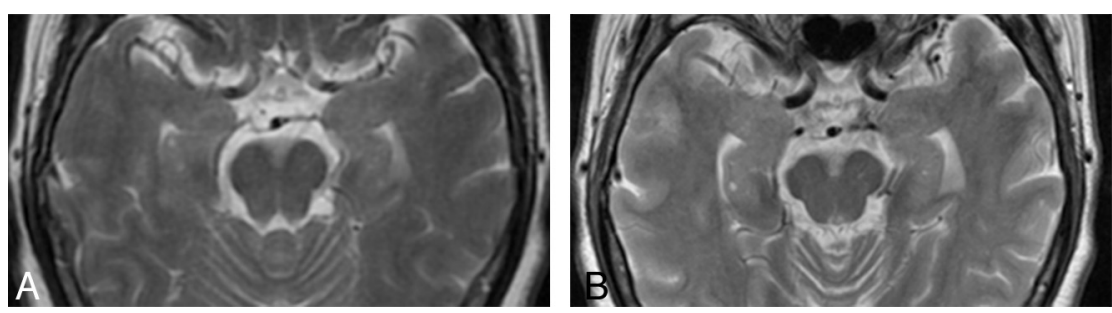

FIG 1. Atrophic change of the hippocampus and cerebral cortex in a 61-year-old woman after $C O$ poisoning. When compared with initial T2-weighted imaging in the acute phase $(A)$, imaging at 3 months after CO inhalation demonstrates enlargement of the bilateral inferior horns of the lateral ventricles and expansion of the cerebral sulci, suggesting atrophy of the hippocampus and cerebral cortex $(B)$. Recent memory disturbance was recovered, but persistent chronic symptoms including slow movement and personality change remained at 3 months after $\mathrm{CO}$ inhalation. elevated nitrite levels and causing subsequent injuries to the cerebral cortex. ${ }^{11}$ Furthermore, hypoxia in the brain causes oxidative stress, cellular necrosis, apoptosis, and ongoing inflammation. ${ }^{12}$

Although it is important to emphasize the damage to the brain that results from hypoxia-related effects, brain damage can also be caused by mechanisms other than hypoxia. CO also combines with cytochrome A and A3 to cause direct poisoning effects, and decreases in levels of cytochrome $\mathrm{C}$ oxidase inhibit mitochondrial metabolism, leading to cellular respiratory dysfunction. ${ }^{13}$ Furthermore, CO-mediated brain damage associated with free radicals has been noticed. CO binds to platelet heme proteins, causing the release of nitric oxide. Excess nitric oxide produces peroxynitrite, impairing mitochondrial function. ${ }^{14}$ As another example, CO causes platelet-to-neutrophil aggregation, and neutrophil degranulation leads to release or production of myeloperoxidase, proteases, and reactive oxygen species, which cause oxidative stress, lipid peroxidation, and apoptosis. ${ }^{14,15}$ Lipid peroxidation alters the structure of MBP, triggering lymphocytic immunologic responses and increasing the activation of microglia. Immunologic responses to altered $\mathrm{MBP}$ cause progressive demyelination accompanied with ongoing inflammation in the CWM. ${ }^{4}$

\section{CONVENTIONAL MR IMAGING}

As mentioned in the previous section, pathologic changes induced by complicated mechanisms progress in the brain from the time of $\mathrm{CO}$ inhalation to even some years later. The timing of MR imaging will thus have a major effect on the results of imaging. ${ }^{16}$ In this review, times between $\mathrm{CO}$ inhalation and MR imaging were strictly defined as the ultra-acute phase within 24 hours; the acute phase between 24 hours and 7 days; the subacute phase between 8 and 21 days; and the chronic phase from 22 days and thereafter.

Since the end of the 1980s, findings of damaged brain from CO poisoning by using conventional MR imaging instead of CT have been reported. In those days, most articles were case reports that described findings in the chronic phase and documented typical findings of bilateral hyperintensities in the GP and CWM on T2weighted images. ${ }^{17,18}$ In these reports, the authors speculated that the main pathologic changes depicted on MR imaging were necrosis in the GP and demyelination in the CWM. These suppositions were based on a report of autopsy findings by Lapresle and Fardeau, ${ }^{19}$ which identified 1) necrosis in the GP, 2) demyelination in the CWM, 3) spondylotic changes in the cerebral cortex, and 4) necrosis in the hippocampus. Damage in the GP is frequently seen in patients poisoned with $\mathrm{CO} .{ }^{20}$ In contrast, damage in other basal ganglia such as the caudate nucleus, putamen, and thalamus has not been reported as frequently as that in the GP. ${ }^{21,22}$ Authors have generally adopted 1 of 2 reasons for selective damage to the GP: that this region is easily affected by the hypoxic-hypotension process because of a poor anastomotic blood supply $^{18,21,23}$ or that CO binds directly to heme iron in the GP, which happens to be a brain region with the highest iron content. $^{24-26}$ Furthermore, some reports have described a "pallidoreticular pattern" of damage to the substantia nigra in addition to the GP, another region with high iron content. $^{26,27}$ The GP often demonstrates different findings of hemorrhagic infarction, which match the phase in which MR imaging is performed. ${ }^{23,28-30}$ Because the main pathologic features are edema in the acute phase and necrosis in the chronic phase, the size of the GP lesion may shrink on consecutive evaluations. ${ }^{31}$ Parkinsonism is the most commonly seen symptom in patients with CO exposure. ${ }^{1,32}$ The possibility that damage to the GP relating to the extrapyramidal tract causes parkinsonism is readily apparent, but parkinsonism can also occur in patients without damage to the GP. Pavese et $\mathrm{al}^{32}$ hypothesized that lesions in the CWM containing tracts outputting and/or inputting to the basal ganglia might cause parkinsonism in patients without GP damage.

Regarding gray matter structures other than the GP, many previous reports have described damage to the hippocampus (Fig $1 A,-B)$. Almost all of these reports documented findings of hippocampal atrophy in the chronic phase ( 2 months to 21 years) and patients experiencing severe persistent symptoms. ${ }^{22,33-35}$ On the contrary, a few reports have mentioned damage to the cerebral cortex. $^{30,32,36}$ These studies documented cortical atrophy in patients with persistent symptoms in the chronic phase. Such damage to both the hippocampus and the cerebral cortex might create lesions responsible for persistent symptoms in the chronic phase. In a report regarding hippocampal lesions in the acute phase, 2 of 4 patients had died within a short period. ${ }^{28}$ Hippocampal lesions in the acute phase may be predictive of a very poor prognosis.

Gray matter could be predicted to be more vulnerable to hypoxia from poisoning than WM, as neurons in gray matter show high activity and a high blood demand. However, neurons may be able to tolerate pure hypoxia for longer than they can tolerate ischemia. ${ }^{37}$ Damage to gray matter should be caused by ischemic changes through the hypoxic-hypotension process. Some authors have pointed out that CWM damage is more frequently detected on MR imaging than damage in the gray matter. A prospective study in which 73 patients with CO poisoning were consecutively enrolled without any selection reported that $12 \%$ of these patients showed CWM damage, whereas damage to the basal ganglia was found in only 1 patient. ${ }^{5}$ CWM has been recognized to be more responsible for chronic symptoms than gray matter. The most common regions of CWM to be affected are the centrum semiovale and periventricular WM. ${ }^{21,28,31,38}$ On T2-weighted images, both lesions are depicted as hyperintense in the bilateral CWM either symmetrically ${ }^{21,39}$ or asymmetrically. ${ }^{36,40}$ The finding of 

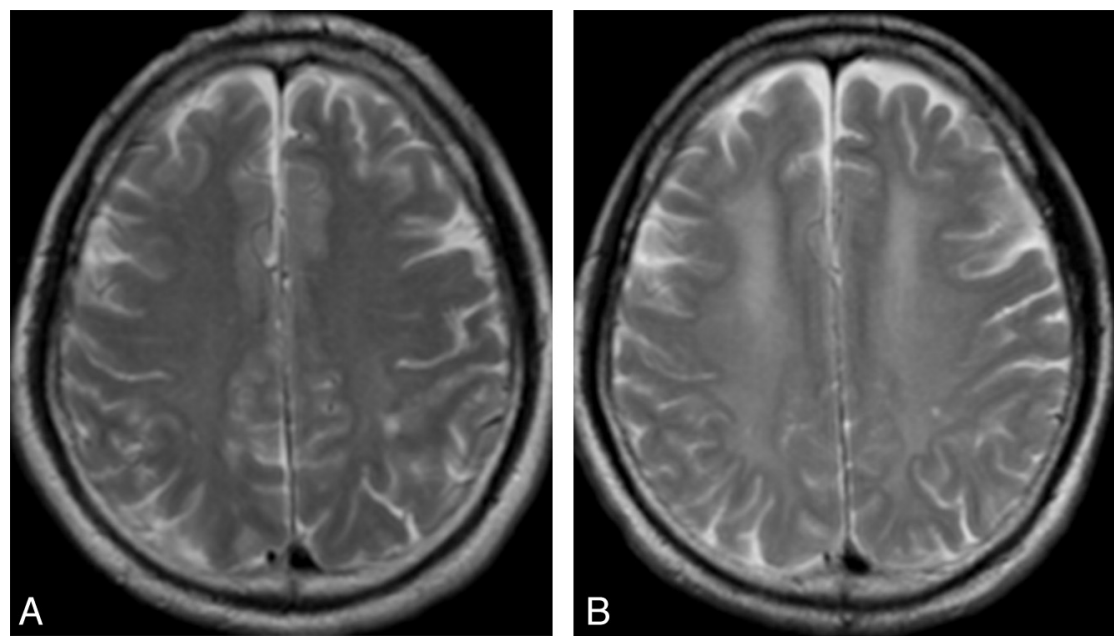

FIG 2. Hyperintensities in the deep WM involving the centrum semiovale in a 53-year-old man with DNS after CO poisoning. A, T2-weighted imaging within 24 hours after $C O$ inhalation reveals slight hyperintensity in the bilateral deep WM and shows a necrotic focus in the left parietal lobe. $B$, At 2 days after the occurrence of DNS, hyperintense areas appear widespread in the bilateral deep WM. Brain atrophy is also apparent in the bilateral frontal lobes.

asymmetric hyperintensity has been speculated to be caused by differences in presumably original arterial blood supply among patients. ${ }^{36}$ Damaged CWM may occasionally show hemosiderindeposit foci suggesting hemorrhagic infarctions or iron extravasation after CO poisoning. ${ }^{41,42}$ CWM damage can be observed in various regions other than the centrum semiovale and periventricular WM, such as the temporal lobe, ${ }^{17,22}$ occipital lobe, ${ }^{17,22}$ parietal lobe, ${ }^{17,43}$ and corpus callosum. ${ }^{38,44}$ However, few reports have provided detailed information regarding damage to the cerebellum and brain stem. This may result from a high tolerance of the posterior structures for hypoxia, and such damage would thus appear under severely hypotensive conditions in addition to hypoxia. $^{28}$ Indeed, patients demonstrating cerebellar damage in the acute or subacute phase uniformly show a poor prognosis. ${ }^{37,39,45}$ Prognosis parallels the amount of damage to WM such as lesions involving the cerebellum. ${ }^{28}$ Consequently, many reports have indicated that the amount of WM damage closely relates to prognosis. ${ }^{6,18,36,46}$ Specific regions in the CWM corresponding to each symptom have also been investigated. Damage to the frontal lobe has been considered to be associated with apathy, loss of motivation, and mutism. ${ }^{32}$ Some prospective studies have examined the relationship between MR imaging findings and results of neurocognitive tests in the chronic phase after $\mathrm{CO}$ poisoning. Kesler et $\mathrm{al}^{47}$ performed consecutive MR imaging in 69 patients and reported that atrophy of the fornix was already apparent at 2 weeks after poisoning, and was associated with reduced verbal memory at 6 months. Parkinson et $\mathrm{al}^{48}$ performed serial MR imaging and cognitive tests on the day of onset, and 2 weeks and 6 months after poisoning in 72 patients, revealing that damage to the centrum semiovale was closely associated with cognitive dysfunction in all phases. Porter et $\mathrm{al}^{44}$ observed atrophy of the corpus callosum at 6 months after poisoning in 62 patients, but cognitive dysfunction in that study appeared independently of corpus callosum atrophy. As mentioned above, however, the brain is damaged more widely in patients with $\mathrm{CO}$ poisoning who have more severe and varied symptoms. Elucidating the specific regions responsible for each symptom may thus be difficult in patients with severe CO poisoning.

Pathologic findings associated with CWM lesions after CO poisoning have been mainly recognized as demyelination. ${ }^{19}$ CWM lesions can be categorized into 3 groups, albeit with a great deal of overlap among groups: 1) the first is small necrotic foci, 2) the second is widespread necrosis accompanying extensive axon destruction and numerous lipid-laden macrophages, and 3) the third is demyelination with relative preservation of the axon in the deep WM. This third group is most often seen in patients with delayed encephalopathy and so-called biphasic myelinopathy of Grinker. ${ }^{21}$ Differences in the extent of demyelination among patients must have major effects on clinical behaviors. Progressive demyelination has been recognized as a cause of DNS and is considered reversible. Many reports have documented that hyperintense areas in the periventricular area and centrum semiovale on T2weighted imaging are more widespread after the appearance of DNS than before DNS (Fig 2A, -B). ${ }^{31,46,49}$ These findings indicate a process of progressive demyelination while DNS develops. DNS can improve within 1-2 years in approximately $60 \%-$ $70 \%$ of patients. ${ }^{50}$ Some investigators have observed areas of decreased hyperintensity on T2-weighted imaging when DNS improved during the chronic phase..$^{21,43,51}$ These findings suggest that MR imaging can depict real-time changes in remyelination of damaged tract fibers with the relative preservation of axons. The extent of damage to WM fibers exerts a large influence on individual prognosis. However, speculation on the histologic changes associated with WM damage by use of conventional MR imaging has limitations because damaged white mater can be caused by various conditions such as cytotoxic edema, necrosis, vasogenic edema, hemorrhagic infarction, iron deposition, ${ }^{41}$ or even previous ischemic lesions before $\mathrm{CO}$ poisoning, ${ }^{48}$ in addition to demyelination.

\section{DWI AND DTI}

Since the early 2000s, brain damage from CO poisoning has been evaluated by DWI, which objectively and quantitatively indicates the magnitude of water molecule diffusion in tissues. Cytotoxic edema in acute ischemic lesions was demonstrated as areas of signal hyperintensity on DWI, leading to a decreased ADC. In such situations, DWI has also been performed for patients with CO poisoning in the acute or subacute phase (Fig $3 A-D$ ). Two reports have provided findings of DWI in the ultra-acute or acute phase, showing hyperintense areas in WM of bilateral frontal and parietal lobes, and reduced ADC values at 12 hours $^{52}$ and 48 hours ${ }^{43}$ after CO poisoning. In these reports, the authors concluded that DWI could depict cytotoxic edema in the damaged WM more sensitively and earlier than conventional MR imaging. Some reports regarding findings of DWI in the subacute and 

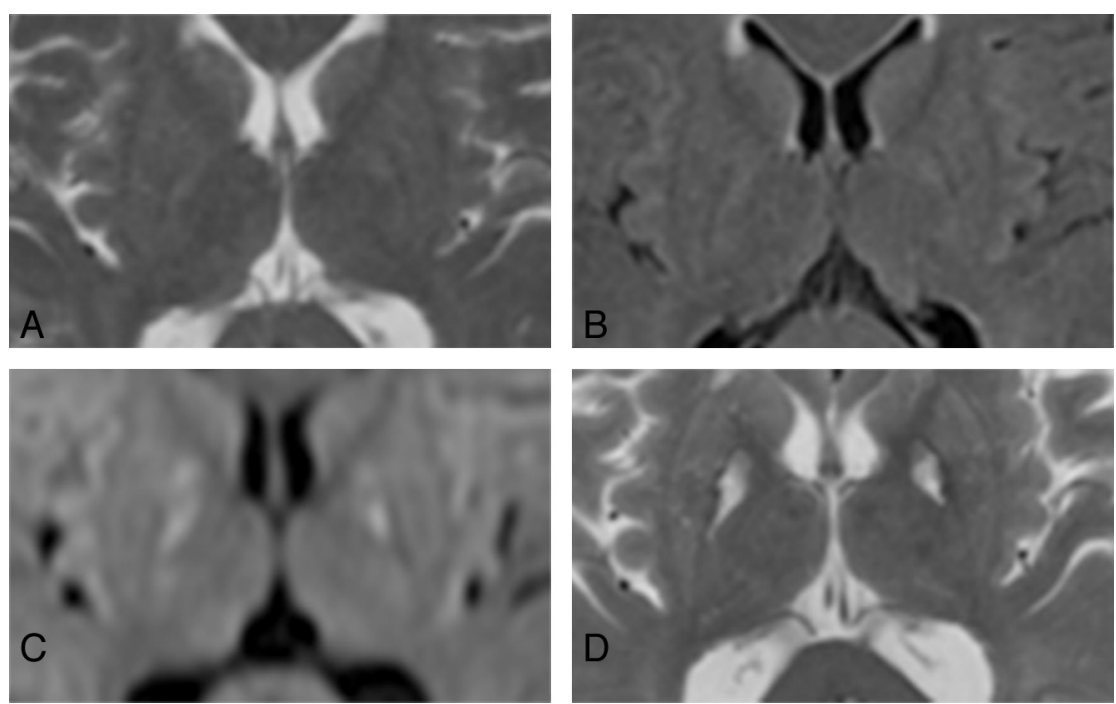

FIG 3. High-sensitivity DWI in the acute phase after CO poisoning in a 64-year-old woman. $A$, T2-weighted imaging; $B$, FLAIR; and C, DWI, all taken within 24 hours after CO inhalation. $D$, T2-weighted imaging at 3 months after CO poisoning. T2-weighted imaging $(A)$ and FLAIR $(B)$ did not demonstrate clear abnormalities in the basal ganglia within 24 hours after $\mathrm{CO}$ poisoning, but markedly high signals were depicted in the bilateral GP on DWI $(C)$ at the same time. Clear hyperintense areas in the bilateral GP were found on T2-weighted imaging after 3 months $(D)$.

chronic phases have been found. ${ }^{25,26,40,53}$ All of these reports documented that areas of signal hyperintensity and low ADC in the CWM remained in the subacute and chronic phases, as in the acute phase. In cases of DNS, ADC after the occurrence of sequelae was lower than before that, and low ADC remained for another 1-2 months. ${ }^{26,40}$ From a pathologic perspective, these findings suggest progressive demyelination with cytotoxic edema. Because these findings of DWI in CO poisoning differ from those in cerebral infarction (ie, low ADC remains for 3-5 days and normalizes within 1-4 weeks after stroke), CWM damage in CO poisoning cannot be explained simply by ischemic change. ${ }^{25,40}$ In a comparison between DWI of the GP and CWM in the subacute phase, the GP appeared hypointense in areas with high $\mathrm{ADC}$, whereas CWM was hyperintense in areas with low ADC. ${ }^{25}$ In that report, the authors concluded that the GP probably undergoes necrosis earlier than CWM; therefore, WM damage would be attributed not only to ischemic changes from the hypoxichypotension process as in GP damage, but also to progressive demyelination. Many reports have found that increases in ADC parallel improvements in symptoms after the appearance of DNS. ${ }^{40,43,53}$ These phenomena might indicate demyelination of myelinated CWM.

Because CWM after CO poisoning shows various histologic changes as mentioned in the previous section, a definite quantitative parameter to specifically indicate demyelination of WM fibers has been desired. DTI, which can show the directionality of water molecule diffusion, and FA as its quantitative value have been recognized to be more suitable than DWI for quantitative evaluation in demyelinating diseases such as multiple sclerosis. DTI has been applied to evaluate CO poisoning since approximately 2005. Most such evaluations have reported low FA values, suggesting a reduction in the directionality of water molecule diffusion because of demyelination, when DTI was performed in the subacute or chronic phases after the appearance of DNS. ${ }^{49,54-56}$ Low FA values might continue until 3 months after poisoning, ${ }^{55}$ and improvement in FA has correlated with improvement in DNS. ${ }^{49,56}$ These changes in FA values should sensitively demonstrate progressive demyelination causing DNS and gradual remyelination while DNS improves. As DNS have been recognized to occur when the extent of demyelination progresses beyond a certain threshold, use of DTI to predict the occurrence of DNS has been suggested. At our institute, FA at the centrum semiovale has been measured at 2 weeks after poisoning in consecutive patients. As a result, we observed that FA in the centrum semiovale had already decreased before the appearance of DNS, and reported the possibility that FA in the subacute phase could offer a predictor for the occurrence of DNS. ${ }^{8}$ The concentration of MBP in CSF, as a marker of demyelinating disease activity, has been proposed as a predictor of DNS. ${ }^{57} \mathrm{An}$ other of our previous studies demonstrated that FA in the centrum semiovale roughly correlates with MBP concentration in the subacute phase (approximately 2 weeks) in patients with CO poisoning who present with chronic persistent symptoms or DNS. ${ }^{58}$ These reports suggest that FA in DTI must be able to sensitively and quantitatively indicate the extent of demyelination after $\mathrm{CO}$ poisoning and, in particular, could demonstrate progressive damage leading to DNS.

Different areas where the region of interest is placed on the CWM might influence the FA values. Lo et $\mathrm{a}^{55}$ reported FA values measured by using regions of interest placed on the centrum semiovale and explained why they placed regions of interest on these areas as follows. All patients in their study showed abnormalities in the deep WM involving the centrum semiovale on T2-weighted imaging, and areas involving the centrum semiovale were relatively homogeneous WM zones related to worse cognitive performance. Selection of these areas was sensible because some reports have shown a relationship between damage in the centrum semiovale and cognitive disorder after $\mathrm{CO}$ poisoning. ${ }^{48,59}$ Our previous study in which we used DTI with voxelbased analysis also showed that deep WM areas including the centrum semiovale were the most widely damaged regions in those patients presenting with chronic symptoms (Fig 4). ${ }^{60}$ When the CO-damaged brain is assessed by DWI or DTI, it is important to emphasize that evaluations of deep WM, including the centrum semiovale, are indispensable.

\section{MR SPECTROSCOPY}

MR spectroscopy enables noninvasive monitoring of changes in metabolism from brain damage in a specified region. Although most previous studies regarding findings of MR spectroscopy in patients with $\mathrm{CO}$ poisoning were only case reports and differences existed in the regions used for placement of the voxel of interest in the brain, these reports have consistently shown increased levels 


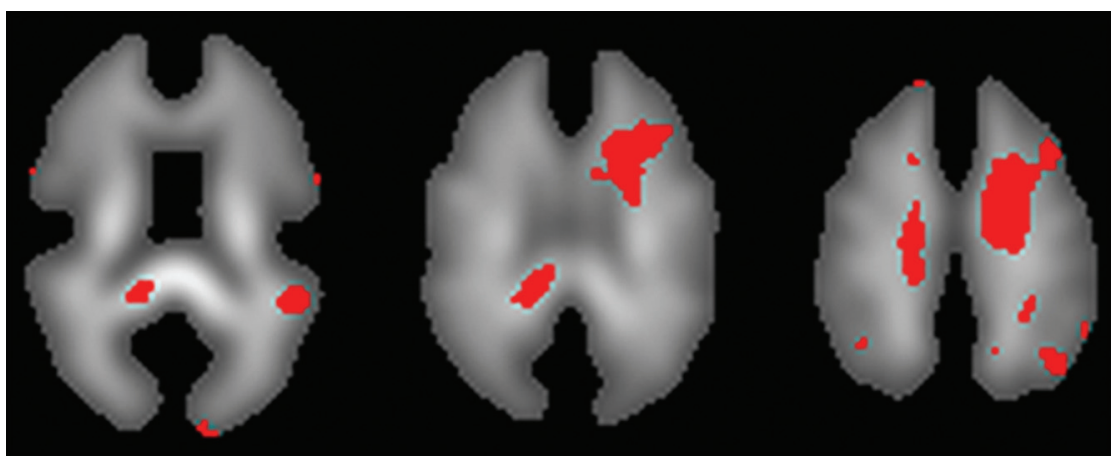

FIG 4. Detection of damaged regions of the CWM by use of voxel-based analysis with DTI. When FA values in all voxels on the FA map were compared voxel by voxel between 2 patient groups (patients with and without chronic neuropsychiatric symptoms), voxels showing a significant difference in FA $(P<.03)$ between groups were identified as reddish voxels on the FA template. The number of reddish voxels was greater in the region corresponding to the deep CWM, including the centrum semiovale, than in other regions.

of Cho indicating active membrane metabolism associated with pathologic conditions such as degeneration and gliosis, decreased NAA reflecting loss or degeneration of axons and/or neurons, and the presence of lactate representing anaerobic glycolysis under ischemic and/or hypoxic conditions. ${ }^{56,61-67}$ These metabolites are usually evaluated by using the relative ratio to $\mathrm{Cr}$ as an indication of stored energy, which reportedly remains relatively stable even in the presence of rapid fluctuations in energy metabolism. When attention is focused on the period in which each metabolite appears, these studies reported that the $\mathrm{Cho} / \mathrm{Cr}$ ratio increased earliest in metabolites and decreased in the late period of the chronic phase, the NAA/Cr ratio began to decrease at the earliest from 3 to 4 weeks, and lactate began to appear at the earliest from 1 to 2 months after $\mathrm{CO}$ poisoning in patients with chronic symptoms. ${ }^{56,61-67}$ It is noteworthy that declines in NAA levels have been proposed as a good prognostic factor, as the NAA/Cr ratio correlates with symptom development, and the presence of lactate acts as a marker of irreversible brain damage because it has been observed in patients with severe chronic symptoms such as akinetic mutism and apallic state. MR spectroscopy findings by $3 \mathrm{~T}$ MR imaging in the subacute phase in 29 patients with CO poisoning found that the $\mathrm{Cho} / \mathrm{Cr}$ ratio in the centrum semiovale was slightly but significantly higher in patients with subsequent chronic-phase symptoms than in patients with transient symptoms in the acute phase. ${ }^{68}$ Increased Cho level in the subacute phase demonstrates inflammation accompanied with progressive demyelination in CWM and may enable prediction of the patient's condition in the chronic phase.

\section{CURRENT AND FUTURE}

So far, most investigations by use of MR imaging and its optional sequences have been rigorously focused on either the GP or CWM as the sites of typical lesions in the chronic phase after CO poisoning. In particular, findings of MR imaging and other sequences on CWM correlating with chronic symptoms allow us to monitor the condition of the patient in the chronic phase. However, brain damage after $\mathrm{CO}$ poisoning should be attributed not only to injury of the CWM, but also to injury of gray matter structures such as the hippocampus or cerebral cortex. In the chronic phase, investigations are needed regarding relationships between damage rosci 2001;13:157-60 to both gray and WM and the severity of symptoms. On the contrary, few reports have described investigations of MR imaging in gray matter structures and CWM in the acute or subacute phase. DTI and MR spectroscopy in the subacute phase have already revealed slight pathologic changes from progressive demyelination starting immediately after $\mathrm{CO}$ inhalation. Interest will eventually migrate to whether findings of MR imaging in the acute phase offer a predictor of the occurrence, severity, and type of chronic symptoms, because DNS can occur as early as within days after $\mathrm{CO}$ inhalation. In our experience of using 3T MR imaging, however, progressively pathologic changes in the acute phase must be too small to observe on conventional MR imaging and even on DWI, DTI, or MR spectroscopy in most patients. In the future, new approaches to the use of MR imaging, such as new sequences or more sensitive imaging such as 7T MR imaging, may reveal and resolve these issues.

Disclosure: Takaaki Beppu-UNRELATED: Grants/Grants Pending: Ministry of Science, Education, Sports and Culture, Japan.* (*Money paid to institution.)

\section{REFERENCES}

1. Choi IS, Cheon HY. Delayed movement disorders after carbon monoxide poisoning. Eur Neurol 1999;42:141-44

2. Weaver LK, Hopkins RO, Elliott G. Carbon monoxide poisoning. N Engl J Med 1999;340:1290; author reply 1292.

3. Weaver LK, Hopkins RO, Chan KJ, et al. Hyperbaric oxygen for acute carbon monoxide poisoning. N Engl J Med 2002;347:1057-67

4. Weaver LK. Clinical practice. Carbon monoxide poisoning. N Engl J Med 2009;360:1217-25

5. Hopkins RO, Fearing MA, Weaver LK, et al. Basal ganglia lesions following carbon monoxide poisoning. Brain Inj 2006;20:273-81

6. Prockop LD, Naidu KA. Brain CT and MRI findings after carbon monoxide toxicity. J Neuroimaging 1999;9:175-81

7. Hurley RA, Hopkins RO, Bigler ED, et al. Applications of functional imaging to carbon monoxide poisoning. J Neuropsychiatry Clin Neu-

8. Beppu T, Nishimoto $H$, Ishigaki $D$, et al. Assessment of damage to cerebral white matter fiber in the subacute phase after carbon monoxide poisoning using fractional anisotropy in diffusion tensor imaging. Neuroradiology 2010;52:735-43

9. Ernst A, Zibrak JD. Carbon monoxide poisoning. $N$ Engl J Med 1998;339:1603-08

10. Okeda R, Funata N, Takano T, et al. The pathogenesis of carbon monoxide encephalopathy in the acute phase-physiological and morphological correlation. Acta Neuropathol 1981;54:1-10

11. Piantadosi CA. Toxicity of carbon monoxide: hemoglobin vs. histotoxic mechanisms. In: Penney DG, ed. Carbon monoxide. Boca Raton, Florida: CRC Press;1996:163-86

12. Piantadosi CA, Zhang J, Levin ED, et al. Apoptosis and delayed neuronal damage after carbon monoxide poisoning in the rat. $\operatorname{Exp} \mathrm{Neu}$ rol 1997;147:103-14

13. Alonso JR, Cardellach F, López S, et al. Carbon monoxide specifically inhibits cytochrome $\mathrm{C}$ oxidase of human mitochondrial respiratory chain. Pharmacol Toxicol 2003;93:142-46

14. Thom SR, Bhopale VM, Han ST, et al. Intravascular neutrophil acAJNR Am J Neuroradiol 35:625-31 Apr 2014 www.ajnr.org 
tivation due to carbon monoxide poisoning. Am J Respir Crit Care Med 2006;174:1239-48

15. Thom SR, Bhopale VM, Fisher D, et al. Delayed neuropathology after carbon monoxide poisoning is immune-mediated. Proc Natl Acad Sci U S A 2004;101:13660-65

16. Hantson P, Duprez T. The value of morphological neuroimaging after acute exposure to toxic substances. Toxicol Rev 2006;25:87-98

17. Horowitz AL, Kaplan R, Sarpel G. Carbon monoxide toxicity: MR imaging in the brain. Radiology 1987;162:787-88

18. Vieregge P, Klostermann W, Blümm RG, et al. Carbon monoxide poisoning: clinical, neurophysiological, and brain imaging observations in acute disease and follow-up. J Neurol 1989;236:478-81

19. Lapresle J, Fardeau M. The central nervous system and carbon monoxide poisoning. II. Anatomical study of brain lesions following intoxication with carbon monoxide (22 cases). Prog Brain Res 1967;24:31-74

20. Sharma P, Eesa M, Scott JN. Toxic and acquired metabolic encephalopathies: MRI appearance. AJR Am J Roentgenol 2009;193: $879-86$

21. Chang KH, Han MH, Kim HS, et al. Delayed encephalopathy after acute carbon monoxide intoxication: MR imaging features and distribution of cerebral white matter lesions. Radiology 1992;184: $117-22$

22. Bruno A, Wagner W, Orrison WW. Clinical outcome and brain MRI four years after carbon monoxide intoxication. Acta Neurol Scand 1993;87:205-09

23. Bianco F, Floris R. MRI appearances consistent with haemorrhagic infarction as an early manifestation of carbon monoxide poisoning. Neuroradiology 1996;38 Suppl 1:S70-72

24. Gandini C, Prockop LD, Butera R, et al. Pallidoreticular-rubral brain damage on magnetic resonance imaging after carbon monoxide poisoning. J Neuroimaging 2002;12:102-03

25. Chu K, Jung KH, Kim HJ, et al. Diffusion-weighted MRI and 99mTcHMPAO SPECT in delayed relapsing type of carbon monoxide poisoning: evidence of delayed cytotoxic edema. Eur Neurol 2004;51:98-103

26. Kinoshita T, Sugihara S, Matsusue E, et al. Pallidoreticular damage in acute carbon monoxide poisoning: diffusion-weighted MR imaging findings. AJNR Am J Neuroradiol 2005;26:1845-48

27. Kawanami T, Kato T, Kurita K, et al. The pallidoreticular pattern of brain damage on MRI in a patient with carbon monoxide poisoning. J Neurol Neurosurg Psychiatry 1998;64:282

28. O'Donnell P, Buxton PJ, Pitkin A, et al. The magnetic resonance imaging appearances of the brain in acute carbon monoxide poisoning. Clin Radiol 2000;55:273-80

29. Yoshii F, Kozuma R, Takahashi W, et al. Magnetic resonance imaging and 11C-N-methylspiperone/positron emission tomography studies in a patient with the interval form of carbon monoxide poisoning. J Neurol Sci 1998;160:87-91

30. Silverman CS, Brenner J, Murtagh FR. Hemorrhagic necrosis and vascular injury in carbon monoxide poisoning: MR demonstration. AJNR Am J Neuroradiol 1993;14:168-70

31. Inagaki $\mathrm{T}$, Ishino $\mathrm{H}$, Seno $\mathrm{H}$, et al. A long-term follow-up study of serial magnetic resonance images in patients with delayed encephalopathy after acute carbon monoxide poisoning. Psychiatry Clin Neurosci 1997;51:421-23

32. Pavese N, Napolitano A, De Iaco G, et al. Clinical outcome and magnetic resonance imaging of carbon monoxide intoxication. A longterm follow-up study. Ital J Neurol Sci 1999;20:171-78

33. Carlesimo GA, Fadda L, Turriziani P, et al. Selective sparing of face learning in a global amnesic patient. J Neurol Neurosurg Psychiatry 2001;71:340-46

34. Gale SD, Hopkins RO, Weaver LK, et al. MRI, quantitative MRI, SPECT, and neuropsychological findings following carbon monoxide poisoning. Brain Inj 1999;13:229-43

35. Henke K, Kroll NE, Behniea H, et al. Memory lost and regained following bilateral hippocampal damage. J Cogn Neurosci 1999;11:682-97
36. Uchino A, Hasuo K, Shida K, et al. MRI of the brain in chronic carbon monoxide poisoning. Neuroradiology 1994;36:399-401

37. Singhal AB, Topcuoglu MA, Koroshetz WJ. Diffusion MRI in three types of anoxic encephalopathy. J Neurol Sci 2002;196:37-40

38. Chalela JA, Wolf RL, Maldjian JA, et al. MRI identification of early white matter injury in anoxic-ischemic encephalopathy. Neurology 2001; $56: 481-85$

39. Durak AC, Coskun A, Yikilmaz A, et al. Magnetic resonance imaging findings in chronic carbon monoxide intoxication. Acta Radiol 2005;46:322-27

40. Kim JH, Chang KH, Song IC, et al. Delayed encephalopathy of acute carbon monoxide intoxication: diffusivity of cerebral white matter lesions. AJNR Am J Neuroradiol 2003;24:1592-97

41. Finelli PF, DiMario FJ Jr. Hemorrhagic infarction in white matter following acute carbon monoxide poisoning. Neurology 2004;63:1102-04

42. Weaver LK, Hopkins RO. Hemorrhagic infarction in white matter following acute carbon monoxide poisoning. Neurology 2005;64: 1101; author reply 1101

43. Teksam M, Casey SO, Michel E, et al. Diffusion-weighted MR imaging findings in carbon monoxide poisoning. Neuroradiology 2002;44:109-13

44. Porter SS, Hopkins RO, Weaver LK, et al. Corpus callosum atrophy and neuropsychological outcome following carbon monoxide poisoning. Arch Clin Neuropsychol 2002;17:195-204

45. Antón M, Alcaraz A, Rey C, et al. Acute hydrocephalus in carbon monoxide poisoning. Acta Paediatr 2000;89:361-64

46. Gotoh M, Kuyama H, Asari S, et al. Sequential changes in MR images of the brain in acute carbon monoxide poisoning. Comput Med Imaging Graph 1993;17:55-59

47. Kesler SR, Hopkins RO, Blatter DD, et al. Verbal memory deficits associated with fornix atrophy in carbon monoxide poisoning. J Int Neuropsychol Soc 2001;7:640-46

48. Parkinson RB, Hopkins RO, Cleavinger HB, et al. White matter hyperintensities and neuropsychological outcome following carbon monoxide poisoning. Neurology 2002;58:1525-32

49. Vila JF, Meli FJ, Serqueira OE, et al. Diffusion tensor magnetic resonance imaging: a promising technique to characterize and track delayed encephalopathy after acute carbon monoxide poisoning. Undersea Hyperb Med 2005;32:151-56

50. Choi IS. Delayed neurologic sequelae in carbon monoxide intoxication. Arch Neurol 1983;40:433-35

51. Yamazaki Y, Yamada A. Delayed encephalopathy after carbon monoxide intoxication. Intern Med 2008;47:1071-72

52. Sener RN. Acute carbon monoxide poisoning: diffusion MR imaging findings. AJNR Am J Neuroradiol 2003;24:1475-77

53. Kim HY, Kim BJ, Moon SY, et al. Serial diffusion-weighted MR imaging in delayed postanoxic encephalopathy. A case study. J Neuroradiol 2002;29:211-15

54. Lin WC, Lu CH, Lee YC, et al. White matter damage in carbon monoxide intoxication assessed in vivo using diffusion tensor MR imaging. AJNR Am J Neuroradiol 2009;30:1248-55

55. Lo CP, Chen SY, Chou MC, et al. Diffusion-tensor MR imaging for evaluation of the efficacy of hyperbaric oxygen therapy in patients with delayed neuropsychiatric syndrome caused by carbon monoxide inhalation. Eur J Neurol 2007;14:777-82

56. Terajima K, Igarashi $\mathrm{H}$, Hirose $M$, et al. Serial assessments of delayed encephalopathy after carbon monoxide poisoning using magnetic resonance spectroscopy and diffusion tensor imaging on 3.0T system. Eur Neurol 2008;59:55-61

57. Kamijo Y, Soma K, Ide T. Recurrent myelin basic protein elevation in cerebrospinal fluid as a predictive marker of delayed encephalopathy after carbon monoxide poisoning. Am J Emerg Med 2007;25:483-85

58. Beppu T, Fujiwara S, Nishimoto H, et al. Fractional anisotropy in the centrum semiovale as a quantitative indicator of cerebral white matter damage in the subacute phase in patients with carbon monoxide poisoning: correlation with the concentration of myelin basic protein in cerebrospinal fluid. J Neurol 2012;259:1698-705 
59. Chang CC, Chang WN, Lui CC, et al. Longitudinal study of carbon monoxide intoxication by diffusion tensor imaging with neuropsychiatric correlation. J Psychiatry Neurosci 2010;35:115-25

60. Fujiwara S, Beppu T, Nishimoto H, et al. Detecting damaged regions of cerebral white matter in the subacute phase after carbon monoxide poisoning using voxel-based analysis with diffusion tensor imaging. Neuroradiology 2012;54:681-89

61. Kado H, Kimura H, Murata T, et al. Carbon monoxide poisoning: two cases of assessment by magnetization transfer ratios and $1 \mathrm{H}-$ MRS for brain damage. Radiat Med 2004;22:190-94

62. Prockop LD. Carbon monoxide brain toxicity: clinical, magnetic resonance imaging, magnetic resonance spectroscopy, and neuropsychological effects in 9 people. J Neuroimaging 2005;15:144-49

63. Kondziella D, Danielsen ER, Hansen K, et al. 1H MR spectroscopy of gray and white matter in carbon monoxide poisoning. J Neurol 2009;256:970-79

64. Murata T, Kimura H, Kado H, et al. Neuronal damage in the interval form of $\mathrm{CO}$ poisoning determined by serial diffusion weighted magnetic resonance imaging plus $1 \mathrm{H}$-magnetic resonance spectroscopy. J Neurol Neurosurg Psychiatry 2001;71:250-53

65. Murata T, Itoh S, Koshino Y, et al. Serial proton magnetic resonance spectroscopy in a patient with the interval form of carbon monoxide poisoning. J Neurol Neurosurg Psychiatry 1995;58:100-03

66. Sakamoto K, Murata T, Omori M, et al. Clinical studies on three cases of the interval form of carbon monoxide poisoning: serial proton magnetic resonance spectroscopy as a prognostic predictor. Psychiatry Res 1998;83:179-92

67. Kamada K, Houkin K, Aoki T, et al. Cerebral metabolic changes in delayed carbon monoxide sequelae studied by proton MR spectroscopy. Neuroradiology 1994;36:104-06

68. Beppu T, Nishimoto H, Fujiwara S, et al. 1H-magnetic resonance spectroscopy indicates damage to cerebral white matter in the subacute phase after CO poisoning. J Neurol Neurosurg Psychiatry 2011; $82: 869-75$ 